\title{
Laparoscopic Cholecystectomy after Upper Abdominal Surgery : Is It Feasible Even after Gastrectomy?
}

\author{
Jungmin Lee, M.D., Jincheol Jeong, M.D., Doojin Kim, M.D., Ph.D., Jooseop Kim, M.D., Ph.D., Taesuk Ryu, M.D. \\ Department of Surgery, Kangdong Sacred Heart Hospital, Hallym University College of Medicine, Seoul, Korea
}

Purpose: Laparoscopic cholecystectomy (LC) is now a standard operation for benign gallbladder (GB) disease. However, previous upper abdominal surgery (UAS) has been regarded as a relative contraindication for LC. The purpose of this study was to examine the effects of history of upper abdominal surgery including gastrectomy on the operative and postoperative results of LC.

Methods: A total of 769 patients underwent LC between March 2008 and December 2015, and the surgical outcomes of 45 patients who had a history of UAS were retrospectively compared with those who did not. Twenty of 45 patients with a history of UAS received gastrectomy, and the remaining 25 received non-gastrectomy UAS. The degree of adhesion and clinical outcomes were further compared between these two groups.

Results: The patients with a history of UAS required placement of a greater number of trocars, longer operation time, longer duration of drainage insertion, and higher open conversion rate (4.4\%) compared to patients with no history of UAS. However, there were no significant differences in postoperative hospital stay or complication rate between the two groups. In the UAS group, $93.3 \%$ of patients required adhesiolysis. There were no significant differences in clinical findings or perioperative outcomes between gastrectomy group and non-gastrectomy group.

Conclusion: A history of UAS including gastrectomy increases the technical difficulty of LC as well as open conversion rate. However, LC can be a feasible and safe approach when performed with adequate methods.

Keywords: Laparoscopic cholecystectomy, Gastrectomy, Adhesion, Conversion to open sugery

This is an Open Access article distributed under the terms of the Creative Commons Attribution Non-Commercial License (http:// creativecommons.org/licenses/by-nc/4.0/) which permits unrestricted non-commercial use, distribution, and reproduction in any medium, provided the original work is properly cited.
Received September 1, 2016

Revised October 25, 2016

Accepted October 27, 2016

Corresponding author

Doojin Kim

Department of Surgery, Kangdong

Sacred Heart Hospital, Hallym

University College of Medicine, 150

Seongan-ro, Seoul 05355, Korea

Tel: +82-2-2224-2222

Fax: +82-2-2224-2570

E-mail: drkdj@hallym.or.kr

\section{INTRODUCTION}

The clinical advantage of laparoscopic cholecystectomy (LC) has been clearly demonstrated compared to open cholecystectomy. Currently, it is widely used as the standard operation for benign gallbladder (GB) disease that requires cholecystectomy. ${ }^{1,2}$ A history of previous upper abdominal surgery (UAS) has been regarded as a relative contraindication for LC. ${ }^{3}$ However, as this field has continued to advance, there have been reports suggesting that LC can be safely performed even in patients who underwent prior UAS. ${ }^{4-6}$

Past studies have encouraged authors to perform LC in most patients who underwent prior UAS. Past studies have demonstrated the safety of LC in patients who underwent prior UAS, and this approach is generally used in patients with a UAS history. However, in the case of gastrectomy, there is a deformation of the anatomical structure of the gastroduodenal area that could increase the risk of LC. In addition, to the best 
of our knowledge, no previous studies have reported the effect of different types of previous gastric surgery on LC outcomes. In the present study, the results of LC were compared between patients who previously underwent gastrectomy and those who underwent other UAS.

\section{MATERIALS AND METHODS}

Laparoscopic approach was primarily performed on patients who required cholecystectomy by targeting all the patients who underwent UAS since 2008. Consent to participate in the study was granted by patients after they were provided with information on the advantages of LC as well as the possibility of injury to other organs and conversion to open laparotomy due to adhesion caused by the previous operation and deformation of anatomical structure. Among the 769 patients who consecutively underwent LC from April 2008 to December 2015, a total 45 patients including 20 patients who underwent previous gastrectomy and 25 patients who underwent nongastrectomy UAS were enrolled. For patients with gastrectomy, medical records for the type of gastrectomy (partial and total) and the type of reconstruction were confirmed, and if the reconstruction type was unclear, it was confirmed by performing gastroscopy.

\section{Surgical procedure}

LC was performed by an experienced surgeon. Basic LC was performed using the three-port method. First, after the skin was incised via a transverse semicircular incision under the umbilicus, the fascia and peritoneum were opened with an open method, and pneumoperitoneum was created by inserting an 11-mm trocar. Next, under direct visual observation from telescope through the first port in the abdominal cavity, a second $10-\mathrm{mm}$ trocar was placed at the subxiphoid area and a third 5-mm trocar was placed on the midaxillary line of the right upper quadrant of the abdomen.

If the patient underwent a previous UAS, pneumoperitoneum was created by inserting an 11-mm trocar through the fascia and peritoneum using the open technique of vertical incision at an extended line with the upper midline incision ending below the umbilicus. The severity of adhesion in the peritoneal cavity was confirmed by inserting the telescope. After confirming a site where adhesion of the abdominal wall was not present in the right lower abdomen, a second 5-mm trocar was inserted after marking the position by pushing it externally with a fingertip. If there was no free space in the subxiphoid area due to the previous operation, adhesiolysis was attempted using endoscissors through the second 5-mm trocar site. If the patient's omentum and small intestine were adherent to the abdominal wall along the existing upper abdominal incisional scar, then a third $10-\mathrm{mm}$ trocar was inserted in the subxiphoid area after dissecting the site of adherence (Fig. 1A).

If there was an adhesion of the transverse colon or small intestine throughout the entire right upper abdominal cavity and gallbladder, the colon and small intestine were dissected by holding the endograsp and endoscissors with both hands after inserting an additional 5-mm trocar in the left lower abdomen or central site (Fig. 1B). Then, a fourth 10-mm trocar was inserted by securing the subxiphoid area. The cholecystectomy was subsequently performed using the aforementioned operative methods. In patients with severe adhesion, adhesiolysis was successfully performed by inserting a fifth 5-mm trocar as necessary. After the operation was completed, a closed suction drain was indwelled in all patients by using a $5-\mathrm{mm}$ trocar site. The objective of the drain was to detect po-
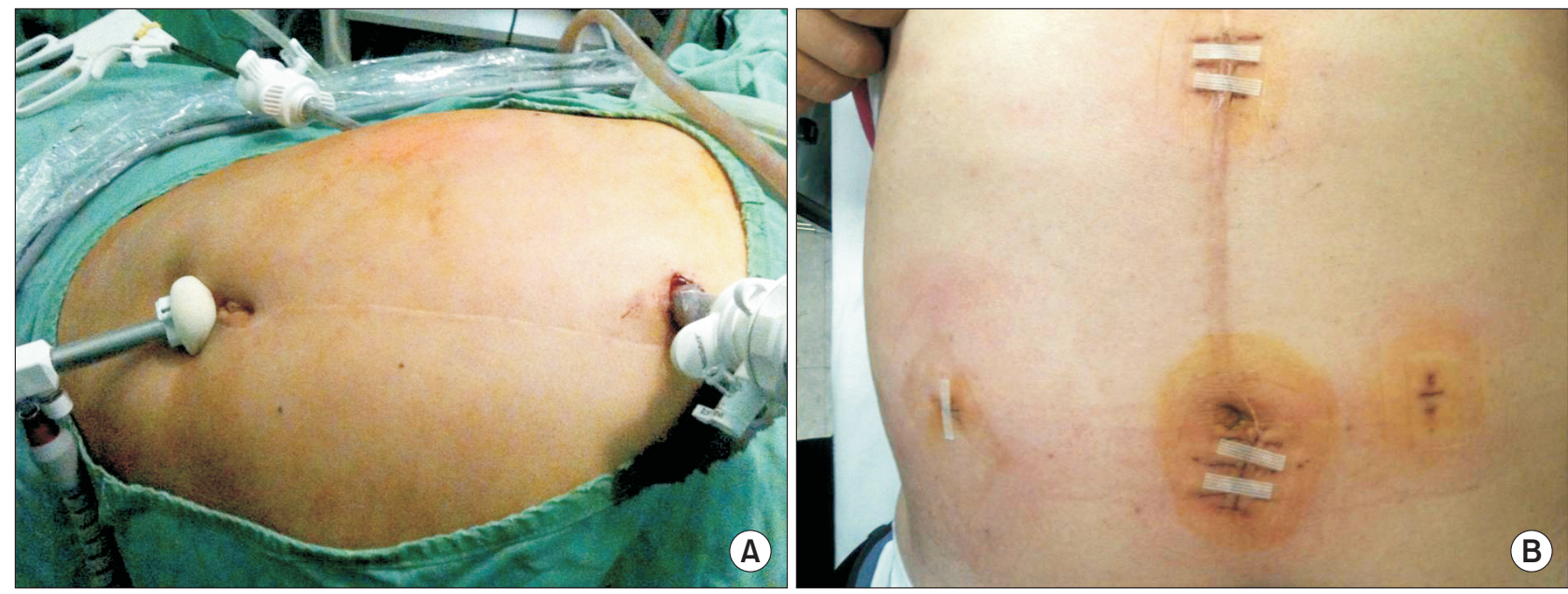

Fig. 1. (A) Location of 3 ports in upper midline incision. (B) Additional $5 \mathrm{~mm}$ port insertion site at left lower abdomen. 
tential organ injury that may have occurred after adhesiolysis. The drain was removed 1 day after operation, if drainage was not turbid with bile or blood and less than $50 \mathrm{cc}$ in volume.

Adhesion was graded depending on its severity. If the gallbladder was visible in the operative field or cholecystectomy progressed only with the simple dissection of the omentum, then it was defined as grade 1 (minimal). Grade 2 (moderate) was applied when adhesiolysis of the organ was required due to adhesion of an internal abdominal organ such as the colon or small intestine. Grade 3 (severe) was applied when the adhesion was too severe to insert the trocar at the conventional site or the trocar for adhesiolysis due to a wide abdominal wall adhesion, and when the laparoscopic approach was impossible without an additional (fourth or fifth) trocar Finally, grade 4 was applied when abdominal trocar insertion was impossible.

The severity of cholecystitis may affect the operation. Regardless of gallstones, gallbladder state without inflammation was defined as grade 1 , acute cholecystitis as grade 2, and gangrenous cholecystitis as grade 3 .

\section{Statistical analysis}

The effect on LC was analyzed by comparing the following variables: age, sex, cholecystitis severity, adhesion degree, preoperative white blood cell count, direct bilirubin level, American Society of Anaesthesiologists (ASA) score, operation time, open conversion, postoperative complications, postoperative frequency of analgesic administration, postoperative hospitalization period, postoperative drain maintenance period, and number of trocar insertions.

Statistical variables were analyzed by using SPSS version 22 (SPSS Inc, Chicago, IL). Continuous variables were compared by independent $t$ test or Mann-Whitney $U$ test, and categorical variables by chi-square or Fisher's exact test. The Spearman test was also carried out to identify any correlations among variables. $p<0.05$ was considered significant.

\section{RESULTS}

\section{Patients}

From April 2008 through December 2015, a total of 769 patients were enrolled in the study; LC was attempted in 724 patients who had no previous UAS (no-history group) and 45 patients who had previous UAS (UAS group; Table 1). Patients' mean age was 55 (range 17 93) years and the overall cohort included and approximately equal number of males and females. However, the UAS group was older (62.6 vs. 54.7 years) and included more males than the no-history group (4:1 vs. 1:1). ASA score, which reflects patients' performance status, was better in the no-history group $(p=0.008)$.

When patients were hospitalized, initial blood tests were performed to evaluate their inflammation status. Mean white blood cell count in the UAS group, 10,775/ $\mu$ 1, was significantly higher than that in the no-history group $(9,063 / \mu 1 ; p=0.021)$. However, there was no significant difference in GB status: 244 patients had symptomatic GB stone without inflammation, 428 patients had acute cholecystitis, and 85 patients had severe gangrenous cholecystitis with GB wall necrosis.

\section{Preoperative diagnosis}

The gallbladder was diagnosed by preoperative USG or spiral CT, considering patients' history, medical record review, physical examination, and laboratory tests. To confirm biliary tract deformation from a previous operation and common bile duct (CBD) stone status, 37 patients in the UAS group and 560 patients in the no-history group received magnetic resonance cholangiopancreatography (MRCP). In addition, 11 patients in the UAS group and 139 patients in the no-history group with cases of suspected CBD stone underwent endoscopic retrograde cholangiopancreatography (ERCP). However, there was also one patient who underwent total gastrectomy with Rouxen-Y and 2 patients who underwent partial gastrectomy with B-II. ERCP was successfully performed in all the patients. At the same time, 10 patients in the UAS group were diagnosed as having CBD stone. In these 10 patients, the $\mathrm{CBD}$ stone was successfully removed, and LC was subsequently performed.

Furthermore, for 7 patients in the UAS group and $78 \mathrm{pa}^{-}$ tients in the no-history group, percutaneous transhepatic gallbladder drainage was performed in advance due to preoperative sepsis and other comorbidities. After the patients recovered from these underlying conditions, an elective operation was performed.

\section{Outcomes}

The number of trocar insertions, operation time, and drain insertion significantly differed between the two groups: 24 (53.3\%) and 678 (93.6\%) patients had 3 trocars inserted for LC $(p<0.001)$, mean duration of operation was $144.2 \pm 56.1$ and $72.6 \pm 43.6$ minutes $(p<0.001)$, and $45(100 \%)$ and $407(56.2 \%)$ patients had a drain bag inserted in UAS and no-history groups, respectively $(p<0.001)$. The drains were kept for an average of 3.3 and 2.2 days in each group $(p=0.004)$.

The duration of antibiotic treatment after LC, postoperative hospital stay, and complication rate showed no statistical difference between the two groups. During the operation, two patients (4.4\%) were converted to open surgery in the UAS group because of adhesion and bowel injury, and two patients 
Table 1. Comparison of upper abdominal surgery (UAS) history and no history group

\begin{tabular}{|c|c|c|c|c|}
\hline & $\begin{array}{c}\text { Total } \\
(\mathrm{n}=769)(\%)\end{array}$ & $\begin{array}{l}\text { UAS history } \\
(n=45)(\%)\end{array}$ & $\begin{array}{l}\text { No history } \\
(\mathrm{n}=724)(\%)\end{array}$ & $p$ value \\
\hline Age (years) & 55.0 & 62.6 & 54.7 & 0.001 \\
\hline Gender (M/F) & $390 / 379$ & $36 / 9$ & $354 / 370$ & 0.000 \\
\hline BMI $\left(\mathrm{kg} / \mathrm{m}^{2}\right)$ & 27.6 & 23.2 & 27.9 & 0.720 \\
\hline ASA score (1/2/3) & 275/447/47 (35.8/58.1/6.1) & 7/31/7 (15.6/68.9/15.6) & $268 / 416 / 40(37 / 57.5 / 5.5)$ & 0.008 \\
\hline WBC $\|\mu\|$ & 9,164 & 10,775 & 9,063 & 0.021 \\
\hline Direct bilirubin (mg/d) & 0.78 & 1.07 & 0.76 & 0.391 \\
\hline GB status & & & & 0.563 \\
\hline Symptomatic GB stone & $244(31.7)$ & $12(26.7)$ & $232(32)$ & \\
\hline Acute cholecystitis & $428(55.7)$ & $26(57.8)$ & 402 (55.5) & \\
\hline Gangrenous cholecystitis & $85(11.1)$ & $7(15.6)$ & $78(10.8)$ & \\
\hline ERCP & $150(19.5)$ & $11(24.4)$ & 139 (19.2) & 0.389 \\
\hline PTGBD & $85(11.1)$ & $7(15.6)$ & $78(10.8)$ & 0.324 \\
\hline CT & $641(83.4)$ & $39(86.7)$ & $602(83.1)$ & 0.539 \\
\hline USG & $405(52.7)$ & $20(44.4)$ & $385(53.2)$ & 0.255 \\
\hline MRCP & $597(77.6)$ & $37(82.2)$ & $560(77.3)$ & 0.446 \\
\hline GB stone & $533(69.3)$ & $36(80)$ & $497(68.6)$ & 0.109 \\
\hline CBD stone & $134(17.4)$ & $10(22.2)$ & $124(17.1)$ & 0.382 \\
\hline \multicolumn{5}{|l|}{ Outcomes } \\
\hline Trocar number 3/4/5 & 702/46/3 (91.3/6.0/0.4) & 24/19/2 (53.3/42.2/4.4) & 678//27/1 (93.6/3.7/0.1) & 0.000 \\
\hline Operation time (minutes) & 76.87 & 144.22 & 72.69 & 0.000 \\
\hline Drain & 452 (58.8) & $45(100)$ & 407 (56.2) & 0.000 \\
\hline Drain duration (days) & 2.29 & 3.33 & 2.23 & 0.004 \\
\hline Pain killer (days) & 4.51 & 4.06 & 4.54 & 0.545 \\
\hline Antibiotics (days) & 5.77 & 6.88 & 5.71 & 0.163 \\
\hline Postoperative hospital stay (days) & 5.54 & 7.15 & 5.44 & 0.068 \\
\hline Complication & $117(15.2)$ & $10(22.2)$ & $107(14.8)$ & 0.177 \\
\hline Open conversion & $4(0.5)$ & $2(4.4)$ & $2(0.2)$ & 0.020 \\
\hline
\end{tabular}

$\mathrm{BMI}=$ body mass index; $\mathrm{ASA}=$ american society of anesthesiologists; $\mathrm{WBC}=$ white blood cell; $\mathrm{GB}=$ gall bladder; $\mathrm{CBD}=$ common bile duct; ERCP

= Endoscopic retrograde cholangiopancreatography; PTGBD = Percutaneous transhepatic gallbladder drainage; $\mathrm{CT}=$ Computer tomography; USG = ultrasonography; MRCP $=$ Magnetic resonance cholangiopancreatography.

$(0.2 \%)$ in the no-history group due to $\mathrm{CBD}$ injury and duodenal injury.

\section{Subgroup analysis}

In the UAS group, two subgroups were created: 20 patients who had gastrectomy and 25 patients who had non-gastrectomy UAS. There were 16 cases of partial gastrectomy (35.6\%) and 4 cases of total gastrectomy (8.8\%). There were 8 cases of primary ulcer closure (17.7\%), 7 cases of small bowel resection $(15.5 \%)$ and 5 cases of hemicolectomy (11.2\%). 15 cases caused by ulcer perforation (33.2\%), 13 cases by gastric cancer (28.9\%), and 7 cases by trauma (15.5\%). The average period between the prior surgery and LC was 12.7 years in the gastrectomy group and 14.0 years in the non-gastrectomy group, and the difference was not statistically significant (Table 2 ). 
Table 2. Characteristics of upper abdominal surgery group

\begin{tabular}{|c|c|c|c|c|}
\hline & Gastrectomy group $(n=20)$ & $(\%)$ & Non-gastrectomy group ( $n=25$ ) & $(\%)$ \\
\hline Operation type & Partial gastrectomy & & Primary closure & $8(17.7)$ \\
\hline \multirow[t]{4}{*}{$45(100)$} & with Billoth-II anastomosis & $11(24.4)$ & Small bowel resection & $7(15.5)$ \\
\hline & Partial gastrectomy & & Hemicolectomy & $5(11.2)$ \\
\hline & with Billoth-I anastomosis & $5(11.2)$ & $\mathrm{Etc}^{*}$ & $5(11.2)$ \\
\hline & Total gastrectomy & $4(8.8)$ & & \\
\hline Cause of operation & Gastric cancer & $13(28.9)$ & Ulcer perforation & $8(17.7)$ \\
\hline \multirow[t]{4}{*}{$45(100)$} & Ulcer perforation & $7(15.5)$ & Trauma & $7(15.5)$ \\
\hline & & & Bowel obstruction & $3(6.8)$ \\
\hline & & & Colon cancer & $3(6.8)$ \\
\hline & & & $\mathrm{Etc}^{+}$ & $4(8.8)$ \\
\hline $\begin{array}{l}\text { Intervals (years) } \\
13.4(0.1 \sim 40)\end{array}$ & $12.7(2 \sim 30)$ & & $14.0(0.1 \sim 40)$ & \\
\hline
\end{tabular}

*Splenectomy, Omental vessel ligation for bleeding control, Gastric pull up, Transduodenal mass excision, Bleeding control for hepatic bleeding. ${ }^{\dagger}$ Small bowel cyst, Ampulla of Vater adenoma, T-colon diverticulitis, Hypopharyngeal cancer.

As shown in Table 3, there were no significant differences between the two groups. More than $90 \%$ of patients in both groups required adhesiolysis. Eighteen patients in the gastrectomy group (90\%) and 24 patients in the non-gastrectomy group (96\%) presented adhesion of grade 2 or higher. One patient in the gastrectomy group was converted to open surgery due to the severity of adhesion, which hindered adhesiolysis, and one patient in the non-gastrectomy group was converted to open surgery due to a small bowel injury that occurred during adhesiolysis. There were no significant differences between The two groups did not significantly differ with regard to Trocar number, operative time, drainage, antibiotics, or analgesics. Complications developed in 5 patients in the gastrectomy group (25\%) and 5 patients in the non-gastrectomy group (20\%). In terms of complications that required surgical intervention, there was one case of intraoperative small bowel perforation in non-gastrectomy group, which was repaired via primary closure after open conversion, and one case of bile leakage in the total gastrectomy group, which was resolved via percutaneous transhepatic biliary drainage. There were three cases of wound infection, which were all treated, as well as cases of pneumonia and acute renal failure, both of which were recovered without sequelae.

\section{DISCUSSION}

LC has been regarded as a standard surgical treatment for benign gallbladder disease. However, as LC complications increase in patients who have undergone previous UAS, UAS history has been regarded as a relative contraindication. ${ }^{3.7}$ In addition, there were higher rates of injury to other organs and open conversion during LC. ${ }^{7.8}$ However, from the late 1990s to early 2000s, methods for placing a trocar while avoiding adhesions were introduced, while laparoscopic equipment and surgeons' experience improved.' ${ }^{9,10}$ Moreover, due to successful application of LC, the open conversion rate of LC in patients with history of gastrectomy ranges from 2.9 3.9\% in previous studies, showing no difference to patients undergoing LC without a history of UAS. Therefore, if cholecystectomy is performed by a skilled surgeon, the procedure can be carried out safely in patients with a history of gastric cancer operation. ${ }^{5,6}$ Furthermore, in the present study, the open conversion among 45 patients with UAS history occurred in only 2 cases (4.4\%), which is comparable to other studies. ${ }^{5,6}$

In the present study, there were no significant differences in hospital stay or complication incidence between the UAS and non-UAS groups. However, patients with a history of UAS had adhesions from previous surgery, which required more trocars and a longer operation time for adhesiolysis. Thus, these outcomes seem to be expected, and also are supported by other studies. ${ }^{6,11}$ There may be different opinions regarding drain insertion, but in the present study, drains were inserted in 100\% of patients with a history of past surgery to detect potential bowel injury. This procedure also lengthened the duration of drain intubation. Based on these findings, previous UAS certainly poses difficulty for LC, but the difficulty is limited to the surgery and does not apply to patient recovery.

There are many studies on LC after gastrectomy or other 
Table 3. Comparison of gastrectomy and non-gastrectomy group

\begin{tabular}{|c|c|c|c|c|}
\hline & Total $(n=45)(\%)$ & Gastrectomy $(n=20)(\%)$ & NG op $(n=25)(\%)$ & $p$ value \\
\hline Age (years) & 62.6 & 65.8 & 60.0 & 0.196 \\
\hline Gender (M/F) & $36 / 9$ & $16 / 4$ & $20 / 5$ & 1.000 \\
\hline BMI $\left(\mathrm{kg} / \mathrm{m}^{2}\right)$ & 23.1 & 23.0 & 23.3 & 0.834 \\
\hline ASA score (1/2/3) & 7/31/7 (15.6/68.9/15.6) & $3 / 13 / 4(15 / 65 / 20)$ & 4/18/3 (16/72/12) & 0.762 \\
\hline Interval (years) & 13.4 & 12.7 & 14.0 & 0.617 \\
\hline WBC $\|\mu\|$ & 10,775 & 10,586 & 10,927 & 0.860 \\
\hline Direct bilirubin (mg/dl) & 1.07 & 0.885 & 1.22 & 0.405 \\
\hline GB status & & & & 0.580 \\
\hline Symptomatic GB stone & $12(26.7)$ & $4(20)$ & $8(32)$ & \\
\hline Acute cholecystitis & $26(57.8)$ & $12(60)$ & $14(56)$ & \\
\hline Gangrenous cholecystitis & $7(15.6)$ & $4(20)$ & $3(12)$ & \\
\hline ERCP & $11(24.4)$ & $4(20)$ & $7(28)$ & 0.535 \\
\hline PTGBD & $7(15.6)$ & $3(15)$ & $4(16)$ & 0.927 \\
\hline CT & $39(86.7)$ & $16(80)$ & 23 (92) & 0.239 \\
\hline USG & $20(44.4)$ & $9(45)$ & $11(44)$ & 0.947 \\
\hline MRCP & $37(82.2)$ & $16(80)$ & $21(84)$ & 0.727 \\
\hline GB stone & $36(80)$ & $17(85)$ & $19(76)$ & 0.453 \\
\hline CBD stone & $10(22.2)$ & $4(20)$ & $6(24)$ & 0.748 \\
\hline Adhesion (grade) & & & & 0.598 \\
\hline Minimal (1) & $3(6.7)$ & $2(10)$ & $1(4)$ & \\
\hline Moderate (2) & $21(46.7)$ & $8(40)$ & 13 (52) & \\
\hline Severe (3) & $20(44.4)$ & $9 / 45$ & $11(44)$ & \\
\hline Impossible (4) & $1(2.2)$ & $1(5)$ & $0(0)$ & \\
\hline \multicolumn{5}{|l|}{ Outcomes } \\
\hline Trocar numbers 3/4/5 & 24/19/2 (53.3/42.2/4.4) & $11 / 8 / 1(55 / 40 / 5)$ & $13 / 11 / 1(52 / 44 / 4)$ & 0.958 \\
\hline Operation time (minutes) & 144.22 & 159.2 & 132.2 & 0.109 \\
\hline Drain duration (days) & 3.33 & 3.5 & 3.2 & 0.526 \\
\hline Pain killer (days) & 4.06 & 4.55 & 3.68 & 0.262 \\
\hline Antibiotics (days) & 6.88 & 7.7 & 6.24 & 0.308 \\
\hline Postoperative hospital stay (days) & 7.15 & 7.2 & 7.12 & 0.949 \\
\hline Complication & $10(22.2)$ & $5(25)$ & $5(20)$ & 0.460 \\
\hline Open conversion & $2(4.4)$ & $1(5)$ & $1(4)$ & 0.872 \\
\hline
\end{tabular}

$\mathrm{BMI}=$ body mass index; $\mathrm{ASA}=$ american society of anesthesiologists; $\mathrm{WBC}=$ white blood cell; $\mathrm{GB}=$ gall bladder; $\mathrm{CBD}=$ common bile duct; $\mathrm{ERCP}$

= Endoscopic retrograde cholangiopancreatography; PTGBD = Percutaneous transhepatic gallbladder drainage; $C T$ = Computer tomography; USG = ultrasonography; MRCP = Magnetic resonance cholangiopancreatography .

UAS. ${ }^{4-6,10-12}$ Patients with a previous gastric operation had higher conversion and complication rates than those with other previous UAS. ${ }^{7.11}$ Based on the assumption that the type of prior surgery may have different effects on the outcome of LC, UAS patients in the present study were divided into those who underwent gastrectomy and those who underwent 
a different UAS for a subgroup analysis. We predicted that a history of gastrectomy would have structurally altered the gastroduodenal ligament area and induced adhesion, rendering dissection more difficult during LC, which would in turn increase the open conversion rate or postoperative complications. However, the gastrectomy group did not significantly differ from the non-gastrectomy group. Regardless of the type of prior surgery, the severity of adhesion influenced the length of operation. In one of two open conversion cases, LC was converted to open surgery because of the difficulty of securing an appropriate space for the laparoscopic surgery due to adhesion severity. The other case was converted to open surgery due to a bowel injury that occurred during adhesiolysis. Thus, both cases of open conversion in the UAS group were related to adhesiolysis. They both occurred during the earlier period of the study, and not a single case of open conversion occurred after 2009. Considering these factors, history of UAS certainly is a factor that increases the risk of open conversion during laparoscopic surgery. However, the severity of adhesion has more influence than the type of prior surgery, which can be overcome by an appropriate laparoscopic approach. ${ }^{13-15}$

Various complications developed, including pneumonia, post-operative ileus, wound infection, voiding difficulty, and delirium, but there were no significant differences between groups. Open conversion occurred in two patients $(0.2 \%)$ in the non-UAS group, due to CBD injury in one case and duodenal perforation during gallbladder dissection caused by severe inflammation in the other. In both cases, the patients recovered without additional treatment through appropriate repair following open conversion.

This study has several limitations. Due to the features of the patients in the surgery group, it was difficult to recruit a sufficient number of cases in a short period, and the study was retrospectively conducted for a relatively long period. Furthermore, there are many limitations to comparing and drawing conclusions regarding the outcomes of LC, owing to surgical methods, length of prior disease, and state of cholecystitis. However, there was little bias pertaining to surgical effects, as the surgeries were performed by one surgeon at a single center, and many prior reports also were unable to recruit an adequate number of patients undergoing laparoscopic surgery with a history of UAS. ${ }^{16}$

In conclusion, an increased open conversion rate and operation time are inevitable consequences of LC in patients with a history of UAS, but an appropriate laparoscopic approach can overcome them. The present findings indicate that gastrectomy does not have a particularly greater influence on the outcome of laparoscopic surgery than other types of UAS, but the severity of adhesion from a prior surgery has more influence on surgical difficulty.

\section{REFERENCES}

1) Schirmer BD, Edge SB, Dix J, Hyser MJ, Hanks JB, Jones RS. Laparoscopic cholecystectomy. Treatment of choice for symptomatic cholelithiasis. Ann Surg 1991;213:665-676; discussion 677.

2) A prospective analysis of 1518 laparoscopic cholecystectomies. The Southern Surgeons Club. N Engl J Med 1991;324:1073-1078.

3) Frazee RC, Roberts JW, Symmonds R, et al. What are the contraindications for laparoscopic cholecystectomy? Am J Surg 1992;164:491-494; discussion 495.

4) Kwon $A H$, Inui $H$, Imamura $A$, Kaibori M, Kamiyama Y. Laparoscopic cholecystectomy and choledocholithotomy in patients with a previous gastrectomy. J Am Coll Surg 2001;193:614-619.

5) Sasaki A, Nakajima J, Nitta H, Obuchi T, Baba S, Wakabayashi G. Laparoscopic cholecystectomy in patients with a history of gastrectomy. Surg Today 2008;38:790-794.

6) Kim J, Cho JN, Joo SH, Kim BS, Lee SM. Multivariable analysis of cholecystectomy after gastrectomy: laparoscopy is a feasible initial approach even in the presence of common bile duct stones or acute cholecystitis. World J Surg 2012;36:638-644.

7) Jorgensen JO, Hunt DR. Laparoscopic cholecystectomy. A prospective analysis of the potential causes of failure. Surg Laparosc Endosc 1993;3:49-53.

8) Miller K, Holbling N, Hutter J, Junger W, Moritz E, Speil T. Laparoscopic cholecystectomy for patients who have had previous abdominal surgery. Surg Endosc 1993;7:400-403.

9) Kumar SS. Laparoscopic cholecystectomy in the densely scarred abdomen. Am Surg 1998;64:1094-1096.

10) Karayiannakis AJ, Polychronidis A, Perente S, Botaitis S, Simopoulos C. Laparoscopic cholecystectomy in patients with previous upper or lower abdominal surgery. Surg Endosc 2004;18:97-101.

11) Fraser SA, Sigman H. Conversion in laparoscopic cholecystectomy after gastric resection: a 15-year review. Can J Surg 2009;52:463466.

12) Akyurek N, Salman B, Irkorucu O, et al. Laparoscopic cholecystectomy in patients with previous abdominal surgery. Jsls 2005;9:178-183.

13) Yu SC, Chen SC, Wang SM, Wei TC. Is previous abdominal surgery a contraindication to laparoscopic cholecystectomy? J Laparoendosc Surg 1994;4:31-35.

14) Wongworawat MD, Aitken DR, Robles AE, Garberoglio C. The impact of prior intra-abdominal surgery on laparoscopic cholecystectomy. Am Surg 1994;60:763-766.

15) Patel M, Smart D. Laparoscopic cholecystectomy and previous abdominal surgery: a safe technique. Aust N Z J Surg 1996;66:309311.

16) Bae YS, Lee SM, Kim JH, Kim BS, Joo SH. Laparoscopic Cholecystectomy in Patients with a History of Upper Abdominal Surgery. J Korean Soc Endosc Laparosc Surg 2009;12:108-112. 